\title{
Quebec Cooperative Study of FRIEDREICH'S ATAXIA
}

\section{Phase Two: Etiological Investigations \\ Part One: Clinical and Biochemical Studies}

\author{
Organized by \\ ANDRE BARBEAU
}

Institutions taking part in this study were: l'Institut de Recherches Cliniques de Montréal; l'Hôpital de l'EnfantJésus, Quebec City; l'Institut de Cardiologie de Québec; le Centre Hospitalier de l'Université Laval; l'Institut de Psychométrie et de Méthodes Quantitatives de Québec; le Centre Hospitalier Universitaire de l'Université de Sherbrooke; l'Institut Armand Frappier, Laval; the University of Arizona Health Sciences Center, Tucson; l'Hôpital Hôtel-Dieu de Montréal; l'Hôpital Sainte-Justine de Montréal; l'Université de Montréal and the Wellesley Hospital, Toronto. 